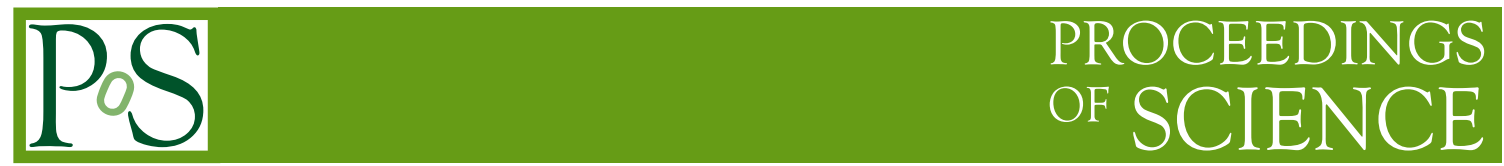

\title{
Recent QCD results from LHC
}

\author{
Katharina Müller*广 \\ University of Zurich \\ E-mail: kmueller@physik.uzh.ch
}

Recent measurements from the ATLAS, CMS and LHCb collaborations are testing QCD with unprecedented precision and in a new energy regime. This talk covers recent measurements by the LHC experiments on the production of jets, isolated photons, electroweak bosons and top quarks.

XIII Quark Confinement and the Hadron Spectrum - Confinement2018

31 July - 6 August 2018

Maynooth University, Ireland

\footnotetext{
* Speaker.

${ }^{\dagger}$ On behalf of the ATLAS, CMS and LHCb collaborations.
} 


\section{Introduction}

Quantum Chromo Dynamics (QCD) is a very successful theory, tested over decades in many experiments. Currently, (next-to-)next-to-leading order (N)NLO calculations exist for most processes, electroweak corrections have been computed and high precision experimental results are available. However, higher-order processes can still be important in some regions of phase space, in particular for multi-parton final states and non-perturbative effects are important, like Parton Distribution Functions (PDF), parton showers and hadronisation.

The LHC has been providing collisions of protons at different centre-of-mass energies, reaching with Run 2 the unprecedented centre of mass energy of $\sqrt{s}$ of $13 \mathrm{TeV}$ in $p p$ collisions. A detailed understanding of QCD is important for almost all physics processes studied by the ATLAS, CMS and LHCb experiments at the LHC and essential for characterising background processes for measurements of and searches for rare or new processes. This paper summarises recent results on the production of jets, isolated photons, top and vector bosons from the LHC experiments. Measurements of multi-parton final states and complex variables, as well as combinations of measurements taken at different energies probe predictions with high precision and provide input for the determination of the PDFs of the proton.

\section{Jet production}

The production of particle jets provides a testing ground for QCD, where jet production is interpreted as the showering of quarks and gluons produced in the scattering process followed by their subsequent hadronisation. At high transverse momenta, $p_{T}$, the scattering of partons can be calculated using perturbative QCD (pQCD). Jet observables, with small theoretical scale uncertainties and reduced jet energy scale uncertainty, can be used to determine the strong coupling constant $\alpha_{s}$.

Analyses using jets at LHC generally use the anti- $\mathrm{k}_{T}$ clustering algorithm [1] with different jet size parameters ranging between 0.4 and 0.7 . Jets of different sizes are affected differently by the impact of the non-perturbative effects of hadronization and the underlying event.

The double-differential di-jet cross-sections measured by ATLAS at $\sqrt{s}=13 \mathrm{TeV}$ divided by the NLO pQCD predictions, corrected for non-perturbative and electroweak effects, are shown in Fig. 1 (left) as a function of the invariant di-jet mass $m_{j j}$ for six bins in rapidity, $y$ [2]. The predictions are consistent with the data over a wide kinematic range in $p_{T}$ between $100 \mathrm{GeV}$ and $3.5 \mathrm{TeV}$ with $|y|<3^{1}$.

A measurement of the double-differential inclusive jet cross-section was performed by CMS as a function of jet $p_{T}$ and $|y|$ and two different distance parameters $\mathrm{R}=0.4$ and 0.7 at $\sqrt{s}=13 \mathrm{TeV}$ in comparison to NLO predictions with different PDF sets[3] (see Fig. 1 (right)). It is observed that jet cross-sections for the larger jet size of $\mathrm{R}=0.7$ are accurately described, while for $\mathrm{R}=0.4$ theory overestimates the cross-section by $5-10 \%$ especially at low $p_{T}$ and central rapidities. This deficit is attributed to missing contributions from parton showers and soft-gluon resummation that are missing in fixed order calculations, consistent with observations at lower centre of mass energies.

\footnotetext{
${ }^{1}$ Natural units with $\mathrm{c}=1$ are used throughout.
} 

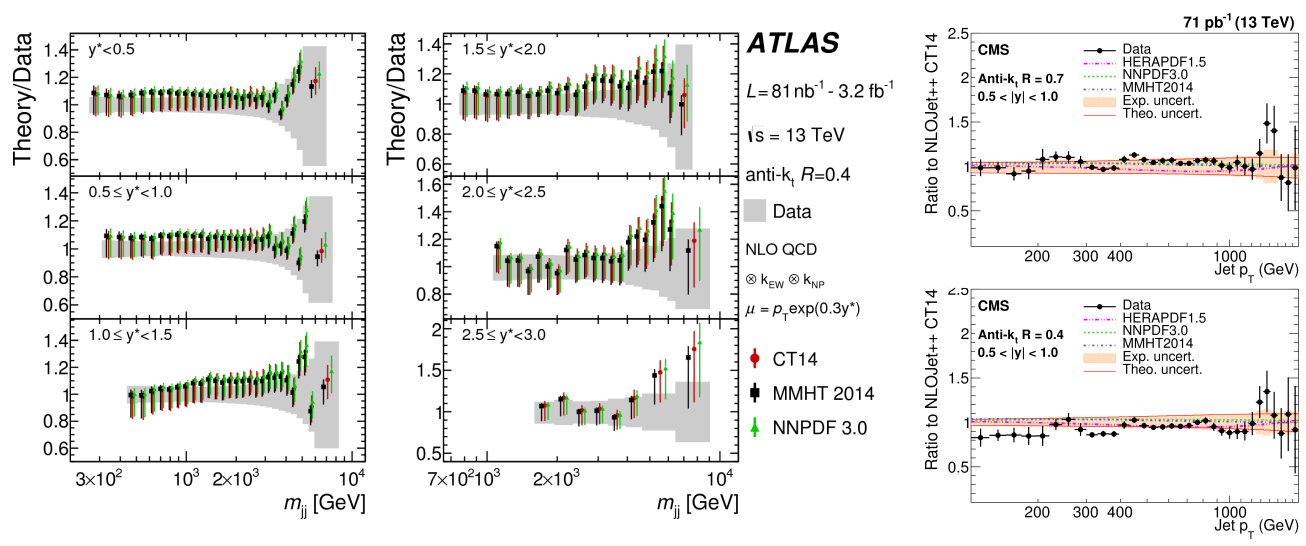

Figure 1: Left: Comparison of the measured di-jet cross-sections with the NLO pQCD predictions shown as the ratios of predictions to the measured cross-sections. The ratios are shown as a function of the invariant di-jet mass, $m_{j j}$, in six $|y|$ bins [2]. Right: Ratio of measured values to theoretical prediction for the inclusive jet cross-section with a distance parameter of $\mathrm{R}=0.7$ (top) and 0.4 (bottom) [3].

Quarks and gluons produced in high-energy particle collisions hadronise before their electric charge can be measured. However, information about the electric charge of the parton that initiated the jet can be estimated by observables that correspond to the momentum-weighted charge sum constructed from charged-particle tracks in the jet, called jet charge $Q$ :

$$
Q^{\kappa}=\frac{1}{P_{T}^{\kappa}(j e t)} \sum_{i} Q^{i}\left(p_{T}^{i}\right)^{\kappa}
$$

where the parameter $\kappa$ controls the relative weight of low and high $p_{T}$ particles.

Figure 2 (left) shows the unfolded leading $p_{T}$ jet charge distribution measured by CMS in comparison to predictions from Powheg [4] plus Pythia 8 [5] [6]. Effects such as multi-parton interaction or initial state radiation do not have a significant effect on the distribution, while disabling final state radiation leads to a significantly broader distribution. Hence the jet charge distribution is mainly sensitive to the modelling of the final state radiation. The variation of the jet charge with leading-jet $p_{T}$ which is sensitive to the quark and gluon jet content in the dijet sample as measured by ATLAS is shown in Fig. 2 (right) for three different values of $\kappa$ [7]. The average charge increases from $0.01 \mathrm{e}$ at $100 \mathrm{GeV}$ to $0.15 \mathrm{e}$ at $1.5 \mathrm{TeV}$ due to the increase of jets initiated by up quarks. The shape is reproduced well by PYTHIA 8 but the prediction tends to be lower than the data.

\section{Strong coupling constant}

Several measurements of $\alpha_{s}$ have already been performed by ATLAS and CMS at different centre of mass energies using jet cross-sections as well as cross-section ratios.

The extraction of $\alpha_{S}$ from jet cross-section data depend on the parametrisation of the PDFs which themselves depend on $\alpha_{S}$ and its scale dependence. This conceptional limitation can be avoided by using ratios of cross-sections in which the PDF dependence cancels to a large extent. 

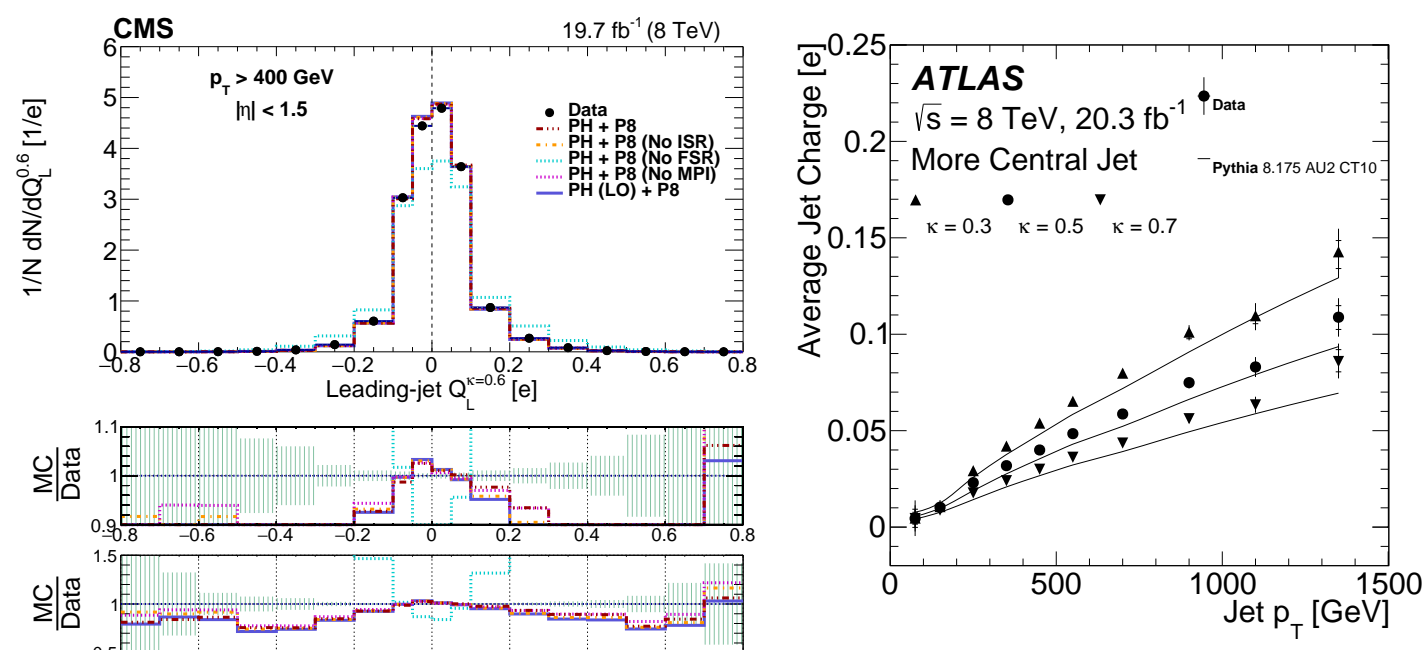

Figure 2: Left: Comparison of the unfolded leading-jet charge distribution with predictions from POWHEG + Pythia 8 (PH+P8). The NLO POWHEG prediction is compared with predictions where initial-state radiation (No ISR), final-state radiation (No FSR), or multiple-parton interactions (No MPI) are disabled in Pythia 8, the LO Powheg prediction (LO) is also shown. [6]. Right: The measured average of the jet charge distribution in units of the positron charge as a function of the jet $p_{T}$ for $\kappa=0.3,0.5$, and 0.7 for central jets compared to the prediction from PYTHIA 8 [7].

ATLAS measured the multi-jet cross-section ratio $R_{\Delta \phi}$, where the quantity $R_{\Delta \phi}$ specifies the fraction of the inclusive dijet events in which the azimuthal opening angle of the two jets with the highest transverse momenta is less than $\Delta \phi_{\max }$ [8]. Figure 3 (left) shows $R_{\Delta \phi}$ as a function of the total transverse momentum $H_{T}$ for different values of $\Delta \phi_{\max }$ and three dijet rapidity intervals $y^{*}$. The $R_{\Delta \phi}$ measurements in the selected kinematic regions are used to determine $\alpha_{S}$ and to test the QCD predictions for its running as a function of the scale $Q=H_{T} / 2$. The individual $\alpha_{S}$ results are compared in Fig. 3 (right) with previously published results obtained from jet measurements and with the prediction by the renormalisation group equation (RGE) [9] using the $\alpha_{S}\left(m_{Z}\right)$ determined from the $R_{\Delta \phi}$ measurement.

\section{Isolated photons}

Measurements using isolated photons and jets are complementary to each other as measurements with photons test pQCD in a cleaner experimental environment and are affected differently by hadronisation and fragmentation effects. However, backgrounds are higher, mainly from jet events with highly energetic $\pi^{0}$ which mimic a single photon. At LHC isolated photons are produced through the dominant leading-order process $q g \rightarrow q \gamma$. The measurements can therefore be used to study the gluon PDF of the proton. In addition, an improved understanding of the production is important as isolated photons are a significant source of background for many searches. Photon production is sensitive to the emission of soft gluons in the initial state and to the nonperturbative fragmentation of quarks and gluons to photons in the final state. Due to this rich phenomenology, theoretical predictions are challenging especially in restricted regions of phase space. 

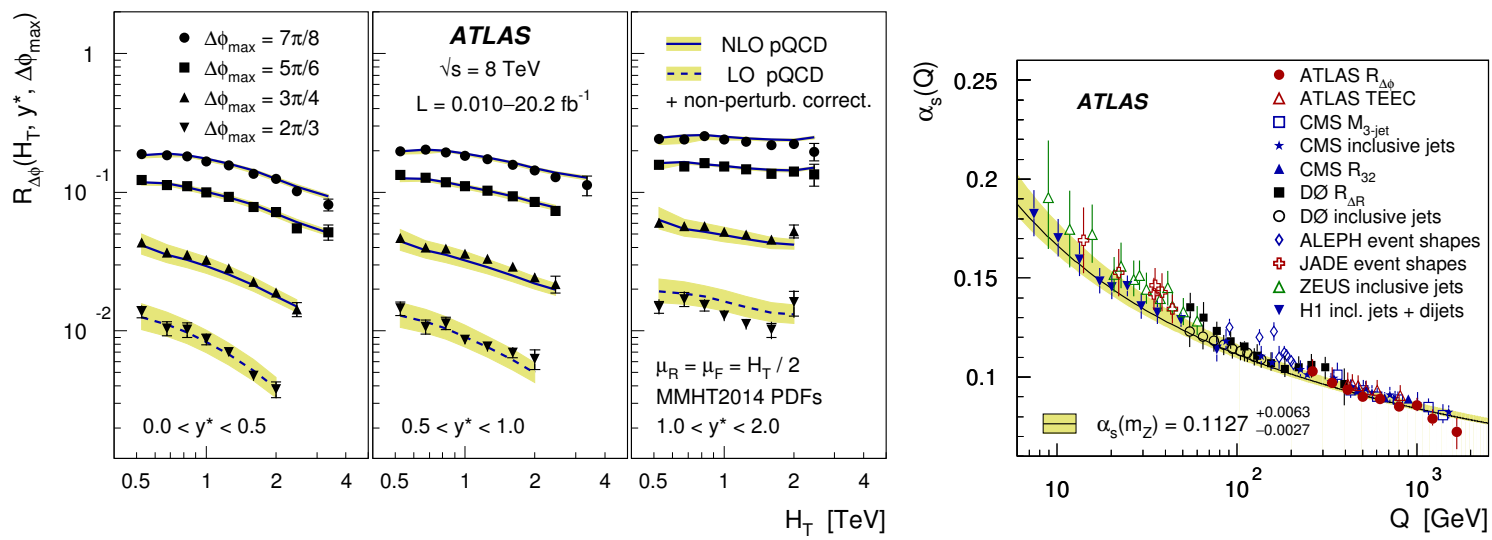

Figure 3: Left: $R_{\Delta \phi}\left(H_{T}, y, \Delta \phi_{\max }\right)$ as a function of $H_{T}$ in three regions of $y$ and for four choices of $\Delta \phi_{\max }$ [8]. Right: The $\alpha_{S}(Q)$ results in the range of $262<Q<1675 \mathrm{GeV}$, compared to the results of previous $\alpha_{S}$ determinations from jet data in other experiments. Also shown is the prediction of the RGE for the $\alpha_{S}\left(m_{Z}\right)$ determined from the $R_{\Delta \phi}$ measurement [8].

The differential cross-sections for inclusive isolated-photon and photon plus jets production at $\sqrt{s}=13 \mathrm{TeV}$ are measured by the CMS and ATLAS experiments. Figure 4 (left) shows the measurement of the photon plus jets production cross-sections as functions of photon transverse energy, $E_{t}$, in different bins of photon and jet rapidities [10]. The measurements are in agreement with theoretical predictions produced using JetPhox [11] NLO calculations. At low to middle range in photon $E_{T}$ the experimental uncertainties are smaller or comparable to theoretical uncertainties, and these measurements provide the potential to further constrain the proton PDFs.
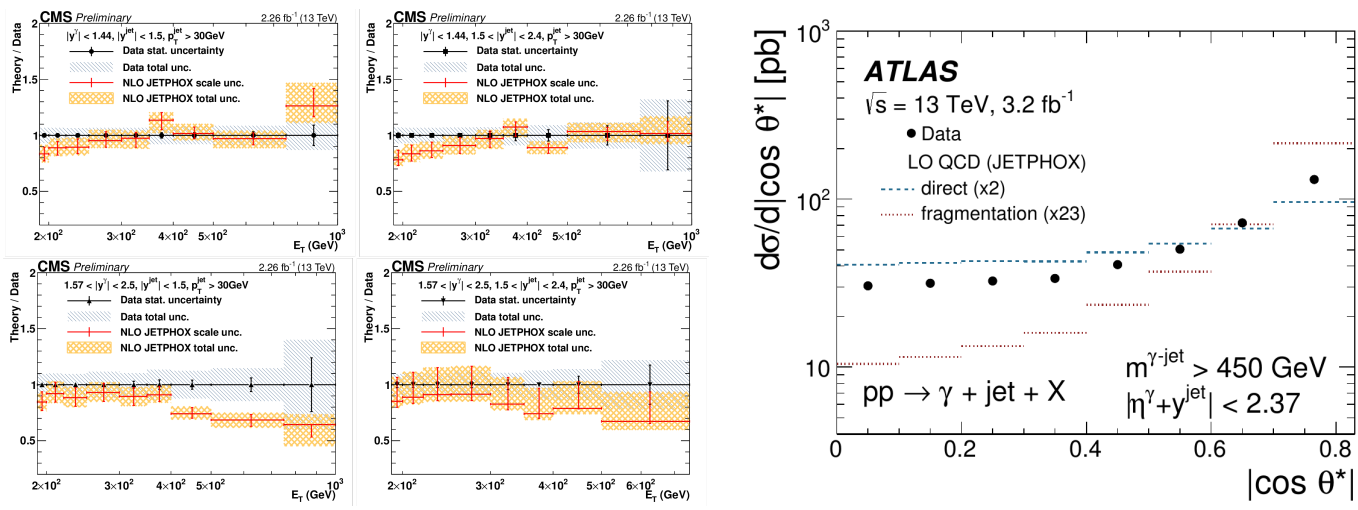

Figure 4: Left: Ratio of theory to data for the differential cross-sections of an isolated photon plus jets as a function of $E_{T}^{\gamma}$ for two different $\left|\eta^{\gamma}\right|$ and $\left|\eta^{\text {jet }}\right|$ regions [10]. The NLO total uncertainty from JETPHOX is displayed as a band. Right: Measured cross-section for isolated-photon plus jet production (dots) as a function of $\left|\cos \theta^{*}\right|$. For comparison, the LO QCD predictions from JETPHOX, normalised to the integrated measured cross-section by the factors shown in parentheses, of direct (dashed) and fragmentation (dotted) processes are shown separately [12]. 

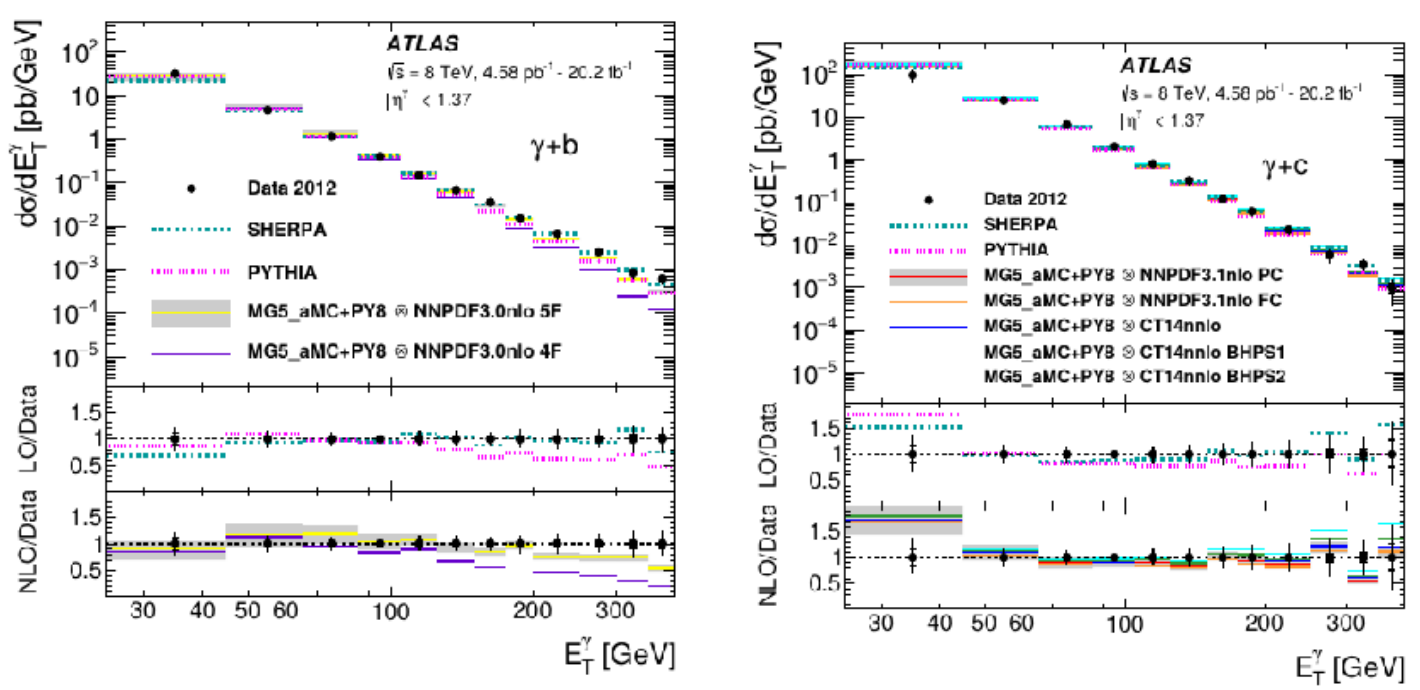

Figure 5: Differential cross-sections for $\gamma+b($ left $)$ and $\gamma+c$ (right) as a function of the photon transverse energy in the central region $\left(\left|\eta^{\gamma}\right|<1.37\right)$ [13].

Two processes contribute to the production of $p p \rightarrow \gamma+$ jet $+X$ events: direct, in which the photon originates from the hard process, and fragmentation, in which the photon arises from the fragmentation of a coloured high $p_{T}$ parton. Measurements of the variable $\theta^{\star}$, where $\cos \theta^{\star}=\tanh (\Delta y / 2)$ and $\Delta y$ is the difference between the rapidities of the photon and the jet can be used to test the interplay of direct and fragmentation processes.

Figure 4 (right) shows the cross-section measured by ATLAS as a function of $\cos \theta^{\star}$ together with the predicted cross-sections from JETPHOX [11] for LO direct and fragmentation processes [12]. Even though the two components are no longer distinguishable at NLO, the LO calculations are useful to illustrate the basic differences in the dynamics of the two processes. The contribution from fragmentation, dominated by gluon exchange, shows a steeper increase as $\left|\cos \theta^{\star}\right| \rightarrow 1$ than that from direct processes, dominated by quark exchange. The shape of the measured cross-section is closer to that of the direct processes than that of fragmentation. This is consistent with the dominance of processes in which the exchanged particle is a quark.

Prompt photons in association with heavy flavour jets can be used to probe the heavy quark content of the proton and test the modelling of heavy flavour production in Monte Carlo (MC). Differential cross-sections of isolated prompt photons in association with a $b-$ or $c$-jet as a function of $E_{T}$, measured by ATLAS at $\sqrt{s}=8 \mathrm{TeV}$, are shown in Fig. 5 [13]. The measured values are compared to LO calculations in PQCD from SHERPA[14] and PYTHIA 8[5] and to NLO calculation from MAD-GRAPH5_AMC@NLO[15] interfaced to PYTHIA 8. For the $\gamma+b$ final state, the best description of the data is provided by the SHERPA predictions. The NLO predictions underestimate the data in the highest $E_{T}$ intervals measured. For the $\gamma+c$ final state, where the measurement uncertainties are larger, all the predictions are in agreement with the data. 

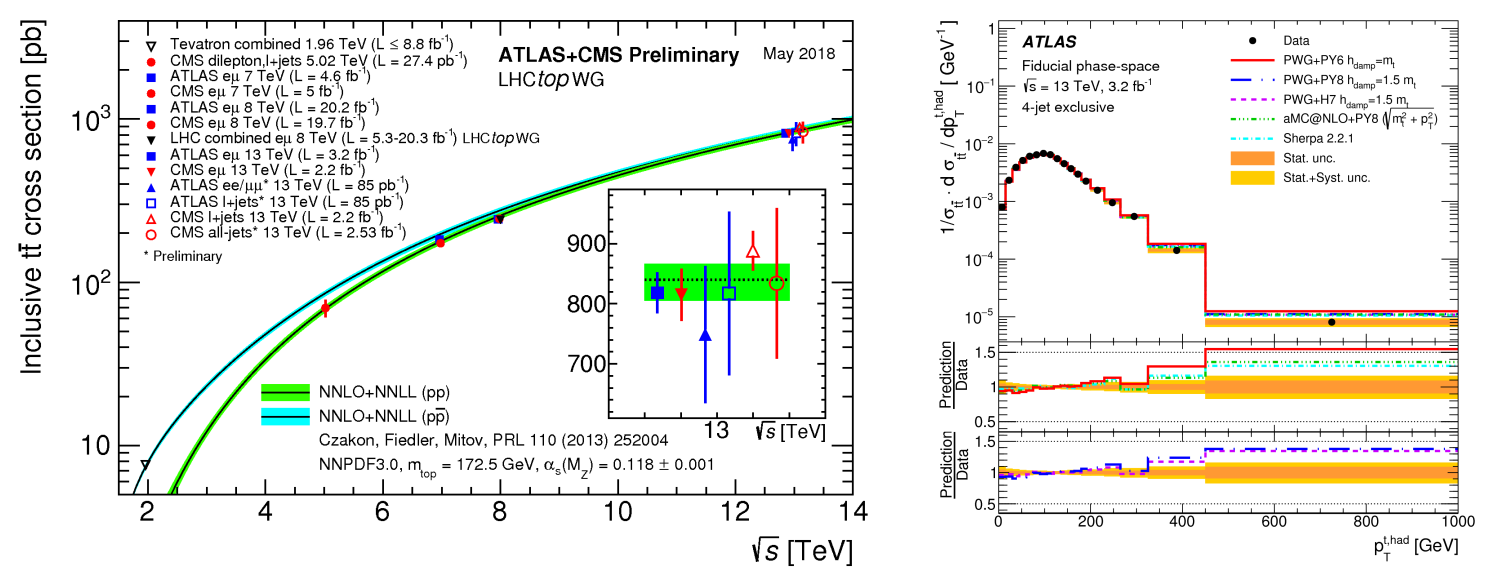

Figure 6: Left: Cross-sections for $t \bar{t}$ production at $p p$ and $p \bar{p}$ colliders at different centre of mass energies [16]. Right: Differential cross-sections for $t \bar{t}$ plus jets production as a function of $p_{T}^{t, h a d}$ in the 4-jet configuration[17].

\section{Top production}

The production of top quarks at hadron colliders represents an important test of the SM with sensitivity to $\alpha_{s}$, the proton PDFs and the mass of the top. The top quark is the heaviest known fundamental particle and its production and decay properties are sensitive to a number of scenarios beyond the SM. Figure 6 shows the measured cross-sections for $t \bar{t}$ production for different centre of mass energies with the latest measurements at $\sqrt{s}=13 \mathrm{TeV}$ from ATLAS and CMS [16]. The measurements are well described by the prediction which is available at NNLO plus next-to-next-to leading $\log$ (NNLL).

The large number of $t \bar{t}$ events produced at LHC allows detailed studies of the characteristics of $t \bar{t}$ production as a function of different kinematic variables. Measurements of differential crosssections for $t \bar{t}$ production in association with jets were studied by ATLAS, these measurements have sensitivity to the effect of gluon radiation. The $t \bar{t}$ events are selected in the lepton (electron or muon) plus jets channel and three mutually exclusive configurations are defined according to the number $(0,1$ or 2$)$ of additional jets reconstructed in each event. Differences between the data and some of the predictions are observed. As an example Fig. 6 (right) shows the measured $p_{T}$ distribution of the top reconstructed in the hadronic channel in the 4-jet configuration. The data is underestimated by the predictions at low values and overestimated at high values; this tendency is reduced at higher jet multiplicity. Overall, the measurements have the potential to further constrain the MC models used to describe the top quark pair production [17].

The unique forward acceptance of the LHCb detector allows measurements in a phase space where top-quark production receives a higher contribution from quark-antiquark annihilation than in the central region and probes higher values of Bjorken- $x$. The higher contribution from quarkinitiated production also results in a larger expected charge asymmetry and thus a better sensitivity 

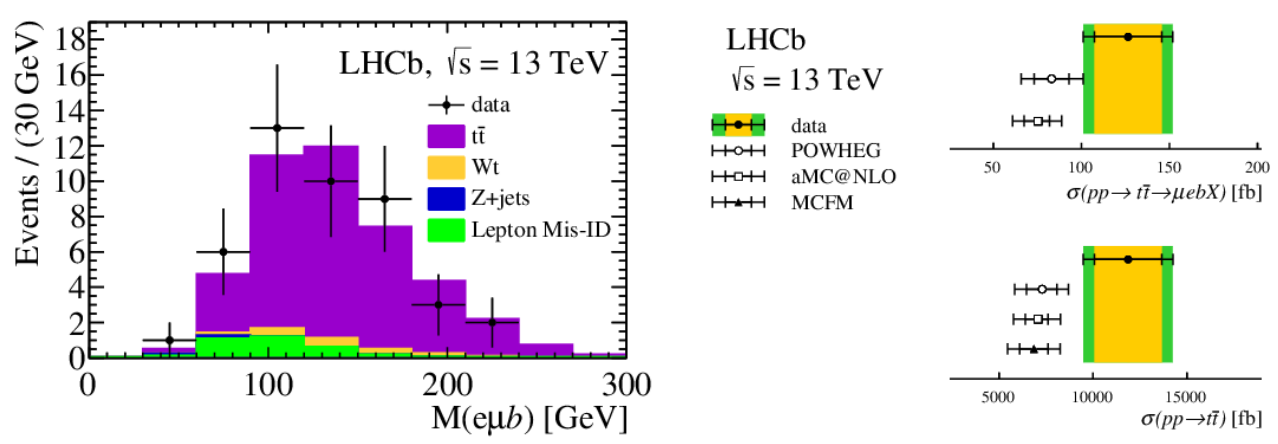

Figure 7: Left: The combined invariant mass of the muon, electron and b-jet forming the top-pair candidates in data compared to the expected contributions from signal and backgrounds. Right: Graphical comparison of the measured cross-sections with the predictions from the AMC@NLO, POWHEG and MCFM generators. For the data, the inner error band represents the statistical uncertainty, and the outer the total, while for the theoretical predictions, the inner band represents the scale uncertainty and the outer represents the total. The prediction is shown (top) for the muon, electron and jet fiducial, and (bottom) for the top quark fiducial region [19].

to contributions from New Physics [18]. LHCb measured the forward top-pair production by requiring an isolated muon, an isolated electron and a $b$-tagged jet. In total 44 candidates were observed with a purity of about $86 \%$. The invariant mass distribution and the cross-section in comparison with the SM predictions are shown in Fig. 7 [19]. The measured cross-section is well in agreement with the predictions from the SM (AMC@ NLO [15], Powheg [4] and MCFM [20]. With the increase in the data-taking capabilities of the LHCb detector in future upgrades, measurements in the $\mu e b$ final state will no longer be statistically limited, and have the potential to achieve the highest precision on the measurement of the $t \bar{t}$ production cross-section at LHCb.

CMS has recently published the first measurement of the underlying event (UE) in $t \bar{t}$ events where the UE is defined as any hadronic activity that cannot be attributed to the particles from the hard scatter. A understanding of the properties of the UE at high energies is mandatory for precision measurements of the top properties and provides a direct test of the universality of the UE at higher energy scales than those probed in minimum bias or Drell-Yan events. This is relevant as a direct probe of colour reconnection, which is needed to confine the initial QCD color charge of the top quark into color-neutral states. In $t \bar{t}$ events the UE contribution is determined to have typically about 20 charged particles (see Fig. 8 (left)) with $p_{T} \simeq p_{z} \simeq 2 \mathrm{GeV}$, vectorially summing to a recoil of about $10 \mathrm{GeV}$. The majority of the distributions analysed indicate a fair agreement between the data and the Powheg +Pythia 8 predictions as illustrated in Fig. 8 (right). There is a negligible dependence on the matrix element generator with which it is interfaced (i.e., PW +PY8 and MG5_aMC). In all distributions the contribution from multi-parton interactions is strong, switching off this component fails to describe the data [21].

\section{Vector bosons}

Events with $W$ and $Z$ bosons decaying into two leptons provide clean experimental signatures 

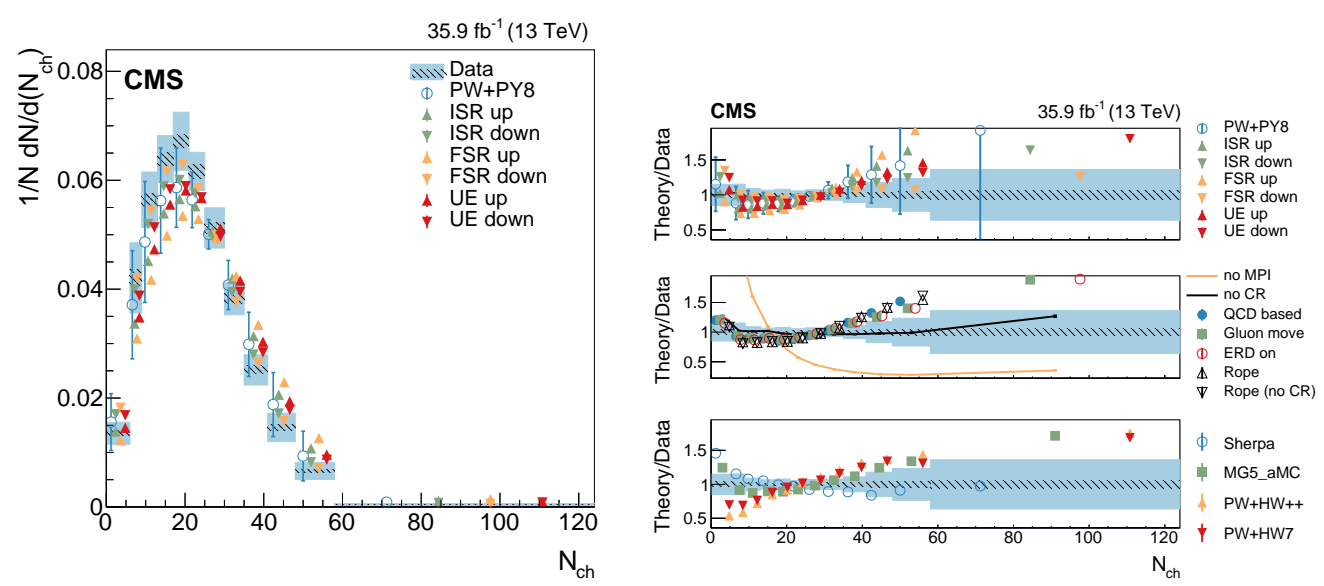

Figure 8: Left: Normalized differential cross-section as a function of the number of charged particles, $N_{c h}$. The data (colored boxes) are compared to the nominal PW+PY 8 predictions. Right: Ratio between models and data with different setups for the simulation[21].

and allow for an efficient selection. Since the cross-sections are known at NNLO with uncertainties at the percent level, measurements with vector bosons provide fundamental input for the determination of PDFs. The NNLO predictions agree with the measurements over the full kinematic range probed by the LHC experiments so far.

The decay $Z \rightarrow b \bar{b}$ provides a standard candle for searches in final states with a $b \bar{b}$ quark pair. The inclusive search for the SM Higgs decay to two $b$ quarks at the LHC is of great interest, since the measurement of the Higgs boson coupling to $b$ quarks is an important test of the SM. Several extensions of the SM predict that new heavy particles that decay to two energetic $b$ quarks could modify the SM cross-section. The measurements of this decay can also be used to demonstrate that no biases are induced by the $b$-jet reconstruction procedure and that the reconstruction efficiencies are evaluated correctly and to determine the $b$-jet energy scale. LHCb has measured $Z \rightarrow b \bar{b}$ in the forward region with the two $b$-tagged jets. A boosted decision tree was used to separate the signal from reduce the contribution from the dominating background from di-jet events and the signal was extracted from a simultaneous fit to the di-jet mass in the signal and the control region [22]. The product of the $Z$-boson production cross-section and the $Z \rightarrow b \bar{b}$ branching fraction in $p p$ collisions at a centre-of-mass energy of $8 \mathrm{TeV}$ is $332 \pm 46$ (stat) \pm 59 (syst) pb in the fiducial region defined by two particle-level $b$-jets with $p_{T}>20 \mathrm{GeV}, 2.2<\eta<4.2$, and $45<m_{j j}<165 \mathrm{GeV}$ in agreement with the SM expectation.

The high centre-of-mass energy at the LHC allows the production of an electroweak boson along with a large number of jets with high transverse momenta. Understanding the kinematics of jets in events with vector bosons associated with several jets is essential for the modelling of backgrounds for other SM processes and searches beyond the SM.

The jet transverse momentum is a fundamental observable of the $Z$ plus jets process and probes pQCD over a wide range of scales. The leading jet $p_{T}$ distribution in inclusive $Z+1,2,3,4$ jet events is shown in Fig. 9 (left) and ranges up to $700 \mathrm{GeV}$. The LO generator (MG5_aMC+Py8 CK$\mathrm{KWL}$ ) models a too-hard jet $p_{T}$ spectrum as already observed at lower centre-of-mass energies. The 

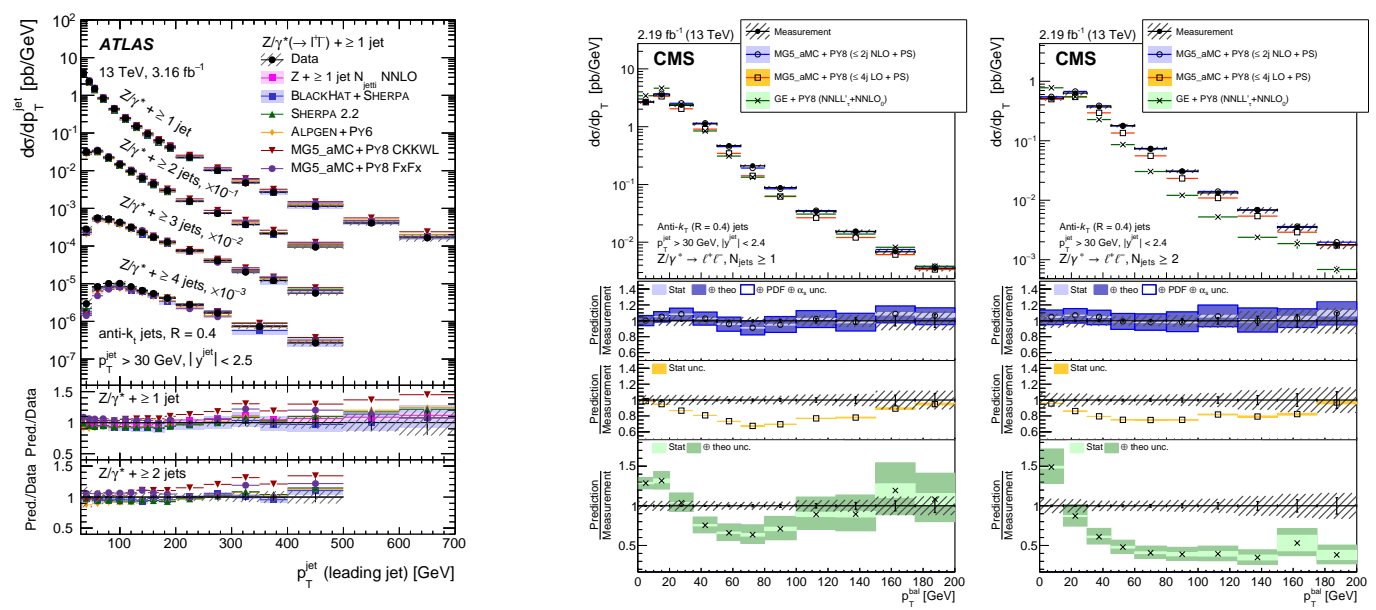

Figure 9: Left: Measured $Z$ plus jets cross-section as a function of the leading jet $p_{T}$ for inclusive $Z$ plus $1,2,3,4$ jets events. The data are compared to various predictions [23]. Right: Measured cross-section for $Z$ plus jets events as a function of the transverse momentum balance, $p_{T}^{b a l}$ between the $Z$ boson and the accompanying jets for events with at least one (left) and two (right) jets [24].

predictions based on NLO matrix elements (BlackHat+Sherpa, Sherpa 2.2, and MG5_aMC+Py8 FxFx), are in agreement with the measured cross-section [23]. Figure 9 (right) shows the transverse momentum balance between the $Z$ boson and the jets for events with at least two jets. This variable is sensitive to additional jets out of the acceptance, as $p_{T}^{b a l}>0$ requires extra activity in the event. While the NLO plus parton showering (PS) prediction gives a good description, LO+PS predicts a too soft distribution. The fixed order (NNLO+NNLL) prediction fails to describe observables sensitive to extra jets mainly due to missing parton showers. The results suggest that multi-parton NLO predictions should be used for the estimation of the $\mathrm{Z}$ plus jets contribution at the LHC in measurements and searches.

Events that involve the production of a vector boson in association with jets originating from heavy flavour (HF) quarks can be used to study specific predictions of the SM and are important to understand as they are backgrounds for many searches such as for example Higgs production in association with a $Z$ boson. Furthermore the cross-section has sensitivity to intrinsic charm (IC) contribution in the proton.

CMS has published a first measurement of $Z$ plus $c$-jets and ratios to $Z$ plus $b$-jets in the central region [25]. The $Z$ boson is identified through its decay into a pair of electrons or muons. Jets with HF quark content are identified through either the semileptonic decay of $c$ or $b$ flavoured hadrons with a muon in the final state, or using exclusive hadronic decays of $D^{ \pm}$or $D^{\star}$ mesons. The extraction of $Z$ plus $c$ and $Z$ plus $b$ event yields is based on template fits to distributions of variables sensitive to the jet flavour. In the semileptonic channel the discriminant is the corrected invariant mass of the particles from the secondary vertex, and for $D$ meson decays a likelihood estimate of the probability that the jet tracks come from the primary vertex. Figure 10 (left) shows the background-subtracted distributions of the corrected secondary vertex mass for the $\mathrm{Z}$ plus jets events with a muon in a jet associated with a secondary vertex. The corrected secondary vertex mass 

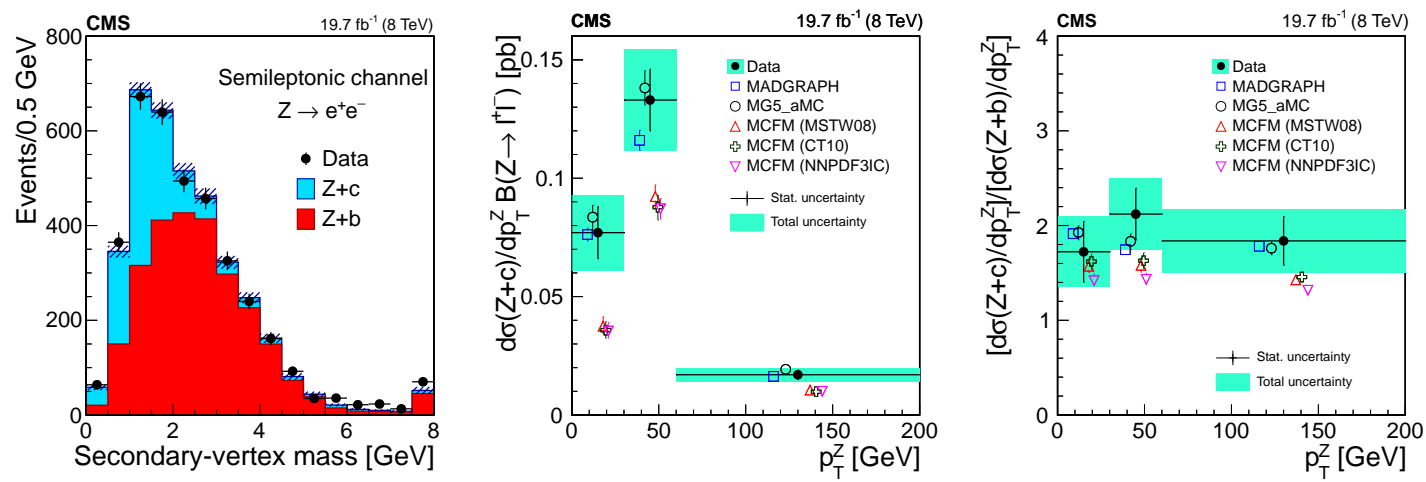

Figure 10: Left: Corrected secondary-vertex mass distributions of candidates with a $Z$ and a $H F$ jet in the di-electron channel for events selected in the semileptonic mode. Middle: Differential $Z+c$ cross section as a function of the transverse momentum of the $Z$ boson. Right: $(Z+c) /(Z+b)$ cross section ratio as a function of the transverse momentum of the $Z$ boson [25].

tends to be larger for $Z$ plus $b$ than for $Z$ plus $c$ events because of the larger mass of the $b$ quark. The differential $Z$ plus $c$ cross section and the ( $Z$ plus $c) /(Z$ plus $b$ ) cross section ratio are shown in Fig. 10 (left) as a function of $p_{T}$ of the $Z$ boson. The two MC generators MADGRAPH[15] (LO) and MG5_AMC (NLO) predictions successfully describe the data while the NLO MCFM [20] predictions with different PDF sets are significantly lower than the data, most pronounced at low $p_{T}$. This deficit can be attributed to missing contributions from parton showers and non-perturbative effects. Measurements in the highest $p_{T}$ region could be sensitive to the existence of an IC component inside the proton. Within the presently still large uncertainties, the measurements of the cross-section and cross-section ratio are consistent with predictions using PDF sets with no IC component.

\section{Summary}

Many new high precision QCD measurements with different final states are available from the LHC experiments ATLAS, CMS and LHCb . These explore regions of phase space where current predictions still struggle to match the data. The combination of measurements taken at different centre of mass energies further allows for more stringent test of the predictions since many uncertainties cancel. A systematic exploration of all the final states and centre of mass energies will largely improve our understanding of QCD, the structure of the proton and the underlying event.

\section{References}

[1] M. Cacciari, G. P. Salam and G. Soyez, JHEP 0804 (2008) 063.

[2] G. Aad et al. [ATLAS Collaboration], JHEP 05 (2018) 195.

[3] V. Khachatryan et al. [CMS Collaboration], Eur. Phys. J. C 76 (2016) no.8, 451 arXiv:1605.04436.

[4] P. Nason, JHEP 11 (2004) 040, arXiv:hep-ph/0409146, S. Frixione, P. Nason, and C. Oleari, JHEP 11 (2007) 070, arXiv:0709.2092, S. Alioli, P. Nason, C. Oleari, and E. Re, JHEP 06 (2010) 043, arXiv:1002.2581. 
[5] T. Sjöstrand, S. Mrenna and P. Z. Skands, Comput. Phys. Commun. 178 (2008) 852, arXiv:0710.3820.

[6] A. M. Sirunyan et al. [CMS Collaboration], JHEP 1710 (2017) 131 arXiv:1706.05868.

[7] G. Aad et al. [ATLAS Collaboration], Phys. Rev. D 93 (2016) no.5, 052003 arXiv:1509.05190. arXiv:1609.05331.

[8] M. Aaboud et al. [ATLAS Collaboration], arXiv:1805.04691.

[9] H. D. Politzer, Phys. Rev. Lett. 30 (1973) 1346, D. J. Gross and F. Wilczek, Phys. Rev. D 8 (1973) 3633.

[10] A. M. Sirunyan et al. [CMS Collaboration], arXiv:1807.00782.

[11] P. Aurenche et al.,Phys. Rev. D 73 (2006) 094007, doi:10.1103/PhysRevD.73.094007,

[12] M. Aaboud et al. [ATLAS Collaboration], Phys. Lett. B 780 (2018) 578 arXiv:1801.00112.

[13] M. Aaboud et al. [ATLAS Collaboration],Phys. Lett. B 776 (2018) 2951710.09560.

[14] T. Gleisberg et al., JHEP 02 (2009) 007 arXiv: 0811.4622 [hep-ph].

[15] J. Alwall et al., JHEP 07 (2014) 079, arXiv: 1405.0301.

[16] https://twiki.cern.ch/twiki/bin/view/LHCPhysics/LHCTopWG

[17] M. Aaboud et al. [ATLAS Collaboration], JHEP 1810 (2018) 1591802.06572.

[18] R. Gauld, Phys. Rev. D91 (2015) 054029, arXiv:1409.8631.

[19] R. Aaij et al. [LHCb Collaboration], JHEP 1808 (2018) 174 arXiv:1803.05188.

[20] J. M. Campbell and R. K. Ellis,Phys. Rev. D60 (1999) 113006, arXiv:hep-ph/9905386, J. M. Campbell, R. K. Ellis, and C. Williams,JHEP 07 (2011) 018, arXiv:1105.0020.

[21] A. M. Sirunyan et al. [CMS Collaboration], arXiv:1807.02810.

[22] R. Aaij et al. [LHCb Collaboration], Phys. Lett. B 776 (2018) 430 arXiv:1709.03458.

[23] M. Aaboud et al. [ATLAS Collaboration], Eur. Phys. J. C 77 (2017) no.6, 361 arXiv:1702.05725.

[24] A. M. Sirunyan et al. [CMS Collaboration], arXiv:1804.05252.

[25] A. M. Sirunyan et al. [CMS Collaboration], Eur. Phys. J. C 78 (2018) no.4, 287 arXiv:1711.02143. 